\title{
Structural brain abnormalities in patients with Parkinson's disease with visual hallucinations: A comparative voxel-based analysis
}

\author{
Romulo Lopes Gama ${ }^{a}$, Veralice Meireles Sales Bruin ${ }^{a, *}$, Daniel Gurgel Fernandes Távora ${ }^{a}$, \\ Fábio L.S. Duran ${ }^{\mathrm{b}}$, Lia Bittencourt ${ }^{\mathrm{c}}$, Sergio Tufik ${ }^{\mathrm{c}}$ \\ a Pós Graduação Ciências Médicas, Universidade Federal do Ceara, Fortaleza, Brazil \\ ${ }^{\mathrm{b}}$ Instituto de Psiquiatria, Hospital das Clínicas, Universidade de São Paulo, Brazil \\ ${ }^{\mathrm{c}}$ Departamento de Psicobiologia, Universidade Federal de São Paulo, Brazil
}

\section{A R T I C L E I N F O}

\section{Article history:}

Accepted 17 March 2014

Available online 13 April 2014

\section{Keywords:}

Visual hallucinations

Parkinson's disease

MRI

Pre-frontal cortex

Operculum

Insula

\begin{abstract}
A B S T R A C T
The objective is to evaluate clinical characteristics and cerebral alterations in Parkinson's disease (PD) patients with diurnal visual hallucinations (VHs). Assessment was performed using magnetic resonance image (MRI) and voxel-based morphometry (VBM). Thirty-nine patients with PD (53.8\%) and ten controls were studied. Voxel based morphology analysis was performed. Eleven patients presented diurnal VHs and among these, six had cognitive dysfunction. Patients with VHs performed worse in the mentationrelated UPDRS I $(p=0.005)$ and motor-related UPDRS III $(p=0.02)$. Patients with VHs showed significant clusters of reduced grey matter volume compared to controls in the left opercula frontal gyrus and left superior frontal gyrus. PD without hallucinations demonstrated reduced grey matter volume in the left superior frontal gyrus compared to controls. Comparisons between patients with VHs regarding the presence of cognitive dysfunction showed that cases with cognitive dysfunction as compared to those without cognitive dysfunction showed significant clusters of reduced grey matter volume in the left opercular frontal gyrus. Cases without cognitive dysfunction had reduced grey matter substance in the left insula and left trigonal frontal gyrus. Judging from our findings, an abnormal frontal cortex, particularly left sided insula, frontal opercular, trigonal frontal gyrus and orbital frontal would make PD patients vulnerable to hallucinations. Compromise of the left operculum distinguished cases with VHs and cognitive dysfunction. Our findings reinforce the theoretical concept of a top-down visual processing in the genesis of VHs in PD.
\end{abstract}

(c) 2014 Elsevier Inc. All rights reserved.

\section{Introduction}

A significant number of patients with Parkinson's disease (PD) will develop visual hallucinations (VHs) in the course of their illness (Rodriguez-Violante, Cervantes-Arriaga, Villar-Velarde, \& Corona, 2010) greatly affecting day care and quality of life (Gomez-Esteban et al., 2011; Naismith \& Lewis, 2011). Visual hallucinations in PD are described as involuntary perceptual experiences in waking state without external visual stimulation and with retained insight (Collerton, Perry, \& McKeith, 2005). Typically, VHs consist of complex visual, commonly moving, images lasting for seconds to minutes, experienced in the alert state with eyes open. It can affect $30-50 \%$ of all PD patients (Meppelink et al., 2009). Disease duration, impairment in activities of daily living and anxiety

* Corresponding author. Address: R. Cel Nunes de Melo 1315, Rodolfo Teófilo, CEP 60.430-270 Fortaleza, Ceará, Brazil. Fax: +55 8532615540.

E-mail address: veralice@superig.com.br (V.M.S. Bruin). have been related to VHs in PD (Gibson et al., 2012). Other associated factors are dopaminergic therapy, cognitive impairment, severity of motor symptoms, sleep disorders and depression (Bar et al., 2006). Possibly, VHs in PD are of multifactorial origin.

Previous neuroimage studies have shown alterations across a number of cortical and sub-cortical brain areas (Ibarretxe-Bilbao, Junque, Marti, \& Tolosa, 2011). The presence of VHs in PD has been related to Lewy body pathology in medial temporal areas (Harding, Broe, \& Halliday, 2002; Williams \& Lees, 2005). Papapetropoulos et al. reported an association between VHs and the presence of Lewy bodies not only in medial and inferior temporal cortices, but also in frontal and parietal areas (Papapetropoulos, McCorquodale, Gonzalez, Jean-Gilles, \& Mash, 2006). Furthermore, Ramirez-Ruiz et al. proposed that structural grey matter reductions in the left lingual gyrus and in the superior parietal lobe, bilaterally in PD patients with VHs, could lead to visuoperceptual impairment; visuoperceptual impairment has been implicated as an important causal factor for the occurrence of $\mathrm{VHs}$ 
(Ramirez-Ruiz et al., 2007). Moreover, the persistent connection between VHs and neuropsychiatric symptoms suggests a common neuropathway (Lewis, Shine, Duffy, Halliday, \& Naismith, 2012). Recently, the pedunculopontine nucleus has been related to VHs in PD: Janzen et al. suggested that the cholinergic system may be important in the causation of VHs (Janzen et al., 2012). Huot et al. demonstrated an increase in the levels of [(3)H]ketanserin binding in the inferolateral temporal cortex of PD patients with VHs suggesting that enhanced 5-HT(2A)-mediated neurotransmission in the inferolateral temporal cortex is associated with the development of VHs in PD (Huot et al., 2010). Taking this evidence together, the dopaminergic, cholinergic and serotoninergic system may all play a role in the development of VHs in PD. Despite this evidence, the pathophysiology of VHs in PD remains to be better clarified.

Attempts to understand visual processing and the complex cerebral connections involved have been made. It has been proposed that visual recognition of complex visual stimuli involves bottom-up visual processing: by this concept visual images are processed from the primary visual cortex to the occipito-temporal cortex (Fenske, Aminoff, Gronau, \& Bar, 2006). Moreover, top-down visual processing also occurs: by this other concept, in suboptimal visual circumstances, a rapid projection of a partially analysed version of the image from visual areas would project to the prefrontal cortex, where it would activate an "initial guess" which is projected back to the temporal cortex (Bar et al., 2006). Such circuitry would generate the false perception of images or VHs in the disease affected brain. How this happen and why PD patients are more vulnerable to VHs than individuals with other parkinsonian syndromes is not completely understood.

Presently, based on previous studies (Goetz, Vaughan, Goldman, \& Stebbins, 2013; Sanchez-Castaneda et al., 2010), we hypothesise that specific brain regions, like frontal cortex, opercular region and insula are related to the presence of VHs in PD. The aim of this study is to investigate clinical characteristics and cerebral alterations, in PD patients, with and without VHs, using magnetic resonance imaging (MRI) and voxel-based morphometry (VBM) analysis.

\section{Materials and methods}

\subsection{Participants}

This is a cross-sectional study involving PD patients recruited from an outpatient unit at a University Hospital in the city of Fortaleza, Brazil. Subjects were selected consecutively from a group of patients being followed in a longitudinal study [Sleep-For-PD study]. Thirty-nine patients fulfilling the diagnostic criteria for PD and clinically confirmed by a specialist (VMSB) were recruited. Evaluations were performed over a period of 12 months (July 2010-July 2011). Specific questionnaires were all measured concurrently in a face-to-face interview by two trained medical staff. Patients were excluded if they had any severe comorbidity and were not competent to provide their informed consent. Controls were recruited from family and friends of medical staff after a complete medical history that excluded, severe comorbidities, depression, mental illness, use of alcohol or other substance. The protocol was analysed and approved by the Ethics Committee (HU-UFC No. 045.0607). All subjects gave written informed consent.

\subsection{Clinical assessments}

Clinical and demographic data, habits and comorbidities were recorded using a standardised questionnaire. All were evaluated with the Folstein MiniMental State Examination (MMSE) (Folstein,
Folstein, \& McHugh, 1975). Cognitive dysfunction was established if MMSE < 23. All cases without cognitive dysfunction were confirmed with the Brazilian version of the Montreal Cognitive Assessment (MoCA cut-off > 25) (Memoria, Yassuda, Nakano, \& Forlenza, 2013). The Levodopa Equivalent Dose (LED) was calculated as: $100 \mathrm{mg}$ of regular levodopa equivalent to $133 \mathrm{mg}$ of controlled released, to $0.33 \times$ levodopa plus entacapone and to $1.0 \mathrm{mg}$ of pramipexole. PD patients were included in the study if they had previously experienced formed VHs. Formed VHs were defined as 'repetitive involuntary images of people, animal or objects that were experienced as real during the waking state but for which there was no objective reality'. The severity of motor and non-motor symptoms was assessed using the Unified Parkinson's Disease Rating Scale (UPDRS) parts I, II, III and IV (Fahn \& Elton, 1987). Patients were evaluated in the "on" phase.

We used the PDSS scale, a 15-item visual analog scale that quantifies several aspects of nocturnal disabilities and sleep problems in PD; this scale has also been validated in Brazil (Margis et al., 2009). A PDSS score $\leqslant 100$ defined troublesome nocturnal symptoms and a cut-off of $<5$ for each item indicated sleep impairment (Tse et al., 2005). Subjective sleep quality was evaluated by the PSQI. The Pittsburgh Sleep Quality Index has seven components, each one, dealing with a major aspect of sleep: subjective quality of sleep, sleep-onset latency, sleep duration, sleep efficiency and the presence of sleep disturbances. Individuals with a total PSQI score greater than five were considered poor sleepers (Buysse, Reynolds, Monk, Berman, \& Kupfer, 1989). Daytime somnolence was assessed by the Epworth Sleepiness Scale (ESS), a questionnaire containing eight items that ask about the expectation of dozing in eight hypothetical situations. An Epworth Sleepiness Scale score of 10 or more indicates excessive daytime sleepiness (Johns, 1991). Both the PSQI (Bertolazi et al., 2011) and ESS (Bertolazi et al., 2009) have been validated in Brazil. Depressive symptoms were evaluated by the Beck Depression Inventory (BDI) and were defined as present if the score was $\geqslant 10$ (Beck \& Steer, 1984; Gorenstein \& Andrade, 1996).

\subsection{MRI protocols and VBM analysis}

Subjects were studied in a 1.5 Tesla GE Signa HDxt scanner (General Eletric, Milwaukee, Wiscosin, USA). A T1-weighted axial volumetric image was acquired using the FSPGR sequence with the following parameters: TR (Repetition Time)=TR $12.5 \mathrm{~ms}$; TI $($ Inversion Time $)=600 \mathrm{~ms} ; \mathrm{TE}($ Echo Time $)=5.1 \mathrm{~ms} ; 1 \mathrm{~mm}$ slice thickness; FOV (field of view) $=25.6 \mathrm{~cm}^{2}$; FA (flip angle) $=12^{\circ}$; matrix $=256 \times 256$; voxel size $=1 \times 0.94 \times 0.94 \mathrm{~mm}$.

Regional differences in grey matter volume were assessed using VBM. The VBM analysis was carried out with SPM8 (Statistical Parametric Mapping) running on Matlab 7 (Mathworks, Natick, Massachusetts, USA). The MRI dataset was oriented manually to place the anterior commissure at the origin of the three dimensional Montreal Neurological Institute (MNI) coordinate system. The images were segmented into grey and white matter partitions using the unified segmentation procedure (Ashburner \& Friston, 2005). The diffeomorphic anatomical registration using exponentiated lie algebra (DARTEL) algorithm (Ashburner, 2007) was used to spatially normalise the segmented image. An additional 'modulation' step consisted of multiplying each spatially normalised grey matter image by its relative volume before and after normalisation; this ensured that the total amount of grey matter in each voxel was preserved. The resulting images were smoothed using a 12-mm full-width at half-maximum (FWHM) isotropic Gaussian kernel. This filtering size was chosen to provide an optimal degree of increment in signal-to-noise ratio and conformation of the MRI data to a normal distribution, thus allowing the use of parametric tests in subsequent statistical comparisons (Mechelli, Friston, 
Frackowiak, \& Price, 2005; Salmond et al., 2002). Between-group statistical comparisons of mean grey matter volumes were performed with the general linear model, based on random Gaussian field theory (Friston et al., 1995). Only voxels with values above an absolute grey matter threshold of .05 entered the analyses. The resulting statistics at each voxel were transformed to $Z$-scores and displayed as SPM maps normalised into standard anatomical space, at the one-tailed $p<0.001$ level of significance (corresponding to a threshold of $Z=3.09$ ). Based on previous literature about grey matter differences in relatives of PD patients, we selected the following a priori regions of interests (ROIs) for the inspection of any significant findings on the SPM ( $t$ ) maps: hippocampus, parahyppocampal gyrus, superior, medium and inferior frontal gyrus, opercula frontal gyrus, trigonal frontal gyrus, insula, supplementar motor area, superior and inferior temporal gyrus (Ibarretxe-Bilbao et al., 2008; Meppelink et al., 2009). The small volume correction (SVC) tool was performed to investigate whether there were significant findings in areas where grey matter had been predicted a priori. The SVC-analyses were reported at family-wise error (FWE) correction for multiple comparisons $(\mathrm{pFWE} \leqslant 0.05)$.

\subsection{Statistical analysis}

Descriptive statistics are presented as mean \pm standard deviation, range and frequency (\% values). ANOVA, followed by post hoc test, when adequate, was used to compare between multiple groups. Fisher's exact test, Mann-Whitney $U$ test for continuous variables and Student's $t$ test for normally distributed data with equal variances were performed to compare between pairs of groups. Statistical analysis was carried out using SPSS for Windows, version 16.0. Statistical significance was set at $p<0.05$.

\section{Results}

Thirty-nine patients with PD and 10 controls were studied. In both groups men were predominant. The mean age of the patients was similar to controls. In this study, 11 PD patients presented with diurnal VHs and among these subjects, six had cognitive dysfunction. In the group of patients without cognitive dysfunction, most were HY stage II $(N=20,71.4 \%)$ followed by stages I $(N=5,17.8 \%)$ and stage III $(N=3,10.7 \%)$. Among patients with cognitive dysfunction, patients were equally distributed in stages II and III $(N=4,36.4 \%)$ followed by stage IV $(N=2$, $18.2 \%)$ and $\mathrm{I}(N=1,9.0 \%)$. All patients used Levodopa and some were on additional monoamino oxidase inhibitors, amantadine and dopaminergic agonists. Almost $1 / 3$ of cases presented dyskinesia. The majority (59\%) referred vivid dreams after interrogation. A history of sudden sleep onset was present in $1 / 3$. Diabetes and arterial hypertension were not different between patients and controls (Table 1 ).

Patients with VHs had more vivid dreams than patients without VHs. Patients with and without VHs had more vivid dreams than control subjects. History of sudden sleep onset was more common in patients with VHs than without VHs. Also, PD patients with VHs performed worse in the mentation-related UPDRS I and motor-related UPDRS III and tended to do worse in the activities of daily living related UPDRS II as compared to those without VHs. Comparison between cases with and without VHs showed that patients with VHs presented worse quality of sleep examined by the PDSS and worse cognitive dysfunction as evaluated by the MMSE. Patients with VHs also presented more depressive symptoms and worse mental dysfunction as compared to control subject (Table 1). Sleep abnormalities as evaluated by the PDSS showed that nocturnal hallucinations were more common in patients with diurnal
VHs and there was also more difficulty falling asleep and night muscle cramps (Table 1). Fifteen patients were found to use sleep medications (38\%) and there was no history of antipsychotics or previous mental disorders or in-hospital care for disturbed behaviour.

Grey matter abnormalities are shown in Table 2 and Fig. 1. Cases with and without VHs were compared to controls. Furthermore, PD patients with $\mathrm{VHs}$ were compared regarding the presence of cognitive dysfunction. As compared to controls, PD patients without VHs showed significant clusters of reduced grey matter volume in the left superior frontal gyrus. As compared to controls, PD patients with VHs showed significant clusters of reduced grey matter volume in the left opercula frontal gyrus and left superior frontal gyrus. Comparisons between patients with VHs regarding the presence of cognitive dysfunction showed that cases with cognitive dysfunction as compared to those without cognitive dysfunction showed significant clusters of reduced grey matter volume in the left opercula frontal gyrus. Cases without cognitive dysfunction had reduced grey matter substance in the left insula and left trigonal frontal gyrus.

\section{Discussion}

Two important findings are reported in this study. First, PD patients, regardless the presence of VHs, have grey matter loss in the left frontal gyrus reflecting cerebral alterations generally associated with PD. In agreement with our results, Kato et al. showed PD patients with excessive daytime sleepiness even without dementia have significant grey matter atrophy in the frontal cortex (Kato et al., 2012). Melzer et al. also showed that marked grey matter atrophy occurs in PD with dementia; however, it is concluded that some grey matter atrophy precedes the development of dementia and may be accelerated once frank dementia begins (Melzer et al., 2012).

The second important findings were that patients with VHs had increased grey matter loss in the opercular part of the inferior frontal gyrus. Also, PD patients with VHs and cognitive dysfunction had more grey matter loss in the operculum. Our results show that the operculum is an important part of the brain network involved in the manifestation of hallucinations and cognitive dysfunction in PD. In agreement with our findings, reduced connectivity involving the left frontal operculum as well as the parietal opercular area has also been identified in patients with schizophrenia and chronic hallucinations (Sommer, Clos, Meijering, Diederen, \& Eickhoff, 2012). Vaden et al. showed that elevated cingulo-opercular activity provides word-recognition benefit showing that the cingulo-opercular network is not only important as a somatosensory area but is also fundamental for cognitive function (Vaden et al., 2013). Of note, hallucinations have been closely connected to cortical dysfunction in PD (Wakamori et al., 2014). Parkinson's disease is related to aging and is accompanied by a decrease in cognitive and motor capacities. In connection with this, Hoffstaedter et al. showed that all brain regions show reduction of grey matter volume with age, however, cognitive and motor capacities, identified as age related functional decline, are specifically associated with grey matter loss in central insula and the adjacent frontal operculum (Hoffstaedter et al., 2014). The occurrence of VHs in PD could occur as part of a cortical release phenomenon and the cinguloopercular network would be an important part of this process as has been claimed in other condition (Adachi, Watanabe, Matsuda, \& Onuma, 2000; Kazui et al., 2009).

Supporting our findings, Ramirez-Ruiz demonstrated that reduced activation of several right prefrontal areas occur in hallucinating PD patients. (Ramirez-Ruiz et al., 2008). Interestingly, hallucinating PD patients as compared with non-hallucinating 
Table 1

Characteristics of Parkinson's disease patients and controls.

\begin{tabular}{|c|c|c|c|c|c|c|}
\hline & Controls $N=10$ & All cases $N=39$ & $p$ Value & Patients without VH (G1) $N=28$ & Patients with VH (G2) $N=11$ & $p$ Value \\
\hline Gender M/F (\%) & $6 / 4(60 / 50)$ & $\begin{array}{l}27 / 12 \\
(69.2 / 30.8)\end{array}$ & $0.45^{\mathrm{a}}$ & $\begin{array}{l}20 / 8 \\
(71.4 / 28.6)\end{array}$ & $\begin{array}{l}6 / 5 \\
(54.5 / 45.5)\end{array}$ & $\begin{array}{l}0.44^{\mathrm{d}} \\
0.41^{\mathrm{e}} \\
0.96^{\mathrm{f}}\end{array}$ \\
\hline Family history of PD Yes/No (\%) & $\begin{array}{l}2 / 7 \\
(22.2 / 77.8)\end{array}$ & $\begin{array}{l}12 / 25 \\
(30.7 / 69.3)\end{array}$ & $0.70^{\mathrm{a}}$ & $\begin{array}{l}10 / 18 \\
(35.8 / 64.2)\end{array}$ & $\begin{array}{l}3 / 8 \\
(27.3 / 72.7)\end{array}$ & $\begin{array}{l}0.72^{\mathrm{d}} \\
0.68^{\mathrm{e}} \\
0.79^{\mathrm{f}}\end{array}$ \\
\hline Age, $y$ Range & $59-78$ & $52-80$ & $0.76^{\mathrm{b}}$ & $52-77$ & $53-80$ & $0.10^{d}$ \\
\hline Mean (SD) & $68.1(7.0)$ & $67.1(8.4)$ & & $65.7(7.8)$ & $70.6(9.1)$ & $\begin{array}{l}0.20^{\mathrm{e}} \\
0.50^{\mathrm{f}}\end{array}$ \\
\hline Age at onset, $y$ Range & - & $34-76$ & - & $34-73$ & $48-76$ & $0.17^{d}$ \\
\hline Mean (SD) & & $59.0(10.4)$ & & $58.0(10.4)$ & $63.1(9.9)$ & - \\
\hline $\begin{array}{l}\text { Disease duration, y Range, } \\
\text { Mean (SD) }\end{array}$ & - & $\begin{array}{l}0-23 \\
6.8(5.3)\end{array}$ & - & $\begin{array}{l}5.3-7.7 \\
6.5(5.0)\end{array}$ & $\begin{array}{l}5.0-9.8 \\
7.4(6.1)\end{array}$ & $\begin{array}{l}0.45^{\mathrm{d}} \\
-\end{array}$ \\
\hline LED mean (SD) & - & $721.8(144.5)$ & - & $723.0(104.8)$ & $716.6(301.3)$ & $\begin{array}{l}0.94^{1} \\
-\end{array}$ \\
\hline Dyskinesia Yes/No (\%) & - & $\begin{array}{l}13 / 26 \\
(30.7 / 69.3)\end{array}$ & - & $\begin{array}{l}9 / 19 \\
(32.2 / 67.8)\end{array}$ & $\begin{array}{l}4 / 7 \\
(36.3 / 63.7)\end{array}$ & $\begin{array}{l}0.51^{\mathrm{d}} \\
-\end{array}$ \\
\hline Vivid dreams Yes/No (\%) & $\begin{array}{l}2 / 7 \\
(22.7 / 77.8)\end{array}$ & $\begin{array}{l}25 / 14 \\
(59.0 / 41.0)\end{array}$ & $0.05^{\mathrm{a}}$ & $\begin{array}{l}13 / 15 \\
(46.4 / 53.6)\end{array}$ & $\begin{array}{l}9 / 2 \\
(81.8 / 18.2)\end{array}$ & $\begin{array}{l}0.04^{\mathrm{d}} \\
0.013^{\mathrm{e}} \\
0.004^{\mathrm{f}}\end{array}$ \\
\hline Sudden sleep onset Yes/No (\%) & - & $14 / 25(33.3 / 66.7)$ & - & $\begin{array}{l}6 / 22 \\
(21.4 / 78.6)\end{array}$ & $\begin{array}{l}8 / 3 \\
(72.7 / 27.3)\end{array}$ & $\begin{array}{l}0.002^{\mathrm{d}} \\
-\end{array}$ \\
\hline Diabetes Yes/No (\%) & $\begin{array}{l}1 / 8 \\
(11.1 / 88.9)\end{array}$ & $\begin{array}{l}7 / 26 \\
(18.0 / 82.0)\end{array}$ & $0.66^{\mathrm{a}}$ & $\begin{array}{l}7 / 21 \\
(75 / 25)\end{array}$ & $\begin{array}{l}2 / 9 \\
(18.2 / 81.8)\end{array}$ & $\begin{array}{l}0.91^{\mathrm{d}} \\
0.64^{\mathrm{e}} \\
0.60^{\mathrm{f}}\end{array}$ \\
\hline Arterial hypertension Yes/No (\%) & $\begin{array}{l}6 / 3 \\
(66.7 / 33.3)\end{array}$ & $\begin{array}{l}18 / 21 \\
(48.7 / 51.3)\end{array}$ & $0.71^{\mathrm{a}}$ & $\begin{array}{l}16 / 12 \\
(57.1 / 42.9)\end{array}$ & $\begin{array}{l}7 / 4 \\
(63.6 / 36.4)\end{array}$ & $\begin{array}{l}0.85^{d} \\
0.70^{e} \\
0.77^{f}\end{array}$ \\
\hline UPDRS I mean (SD) & - & $4.6(3.0)$ & - & $3.1(2.4)$ & $7.7(1.7)$ & $\begin{array}{l}0.000^{* *} \\
-\end{array}$ \\
\hline UPDRS II mean (SD) & - & $16.8(8.8)$ & - & $14.5(7.0)$ & $21.8(10.5)$ & 0.05 \\
\hline UPDRS III mean (SD) & - & $17.7(8.6)$ & - & $15.4(8.3)$ & $22.7(7.4)$ & $\begin{array}{l}0.02^{*} \\
-\end{array}$ \\
\hline UPDRS IV mean (SD) & - & $1-12$ & - & $2.40(2.7)$ & $3.9(3.0)$ & $\begin{array}{l}0.19 \\
-\end{array}$ \\
\hline PDSS mean (SD) & $119.9(18.7)$ & $99.8(25.9)$ & 0.04 & $99.8(25.9)$ & $71.4(19.9)$ & $\begin{array}{l}0.01^{*}, \mathrm{~d} \\
-{ }^{\mathrm{e}} \\
-{ }^{\mathrm{f}}\end{array}$ \\
\hline BDI mean (SD) & $7.6(7.9)$ & $18.1(12.5)$ & $0.04^{\mathrm{c}}$ & $17.5(13.0)$ & $21.9(10.9)$ & $\begin{array}{l}0.07 \\
0.68^{\mathrm{e}} \\
0.001^{\mathrm{f}, * *}\end{array}$ \\
\hline MMSE-Folstein mean (SD) & $25.6(4.3)$ & $25.2(5.3)$ & 0.83 & $25.6(4.3)$ & $19.3(3.7)$ & $\begin{array}{l}0.000^{* *} \\
0.67^{\mathrm{e}} \\
0.02^{\mathrm{f}, *}\end{array}$ \\
\hline
\end{tabular}

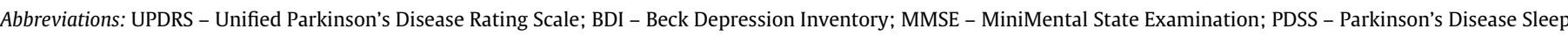
Scale; PSQI - Pittsburgh Sleep Quality Index; ESS - Epworth Sleepiness Scale; RBD - REM Behaviour Disorder.

a Fisher exact test.

b Student's $t$ test.

c Mann-Whitney test.

d G1 versus G2.

e G1 versus control.

f G2 versus control.

${ }^{*} p<0.05$.

** $p<0.01$.

Table 2

List of peaks of most significant differences in group comparisons.

\begin{tabular}{|c|c|c|c|c|c|c|c|}
\hline \multirow[t]{2}{*}{ Groups } & \multirow[t]{2}{*}{ Anatomical location } & \multicolumn{3}{|c|}{ Talairach coordinates } & \multirow[t]{2}{*}{ Cluster dimension } & \multirow[t]{2}{*}{$z$-Score } & \multirow[t]{2}{*}{$p$ Corrected $^{*}$} \\
\hline & & $x$ & $z$ & $y$ & & & \\
\hline \multirow[t]{2}{*}{ PDH versus controls } & Left opercular frontal gyrus & -47 & 11 & -1 & 121 & 4.42 & 0.008 \\
\hline & Left superior frontal gyrus & -18 & 14 & 53 & 121 & 4.12 & 0.023 \\
\hline PDWH versus controls & Left superior frontal gyrus & -18 & 13 & 53 & 1013 & 4.11 & 0.024 \\
\hline $\mathrm{PDH}+\mathrm{CD}$ versus $\mathrm{PDH}-\mathrm{CD}$ & Left opercular frontal gyrus & -56 & 12 & 27 & 146 & 3.59 & 0.027 \\
\hline \multirow[t]{2}{*}{$\mathrm{PDH}-\mathrm{CD}$ versus $\mathrm{PDH}+\mathrm{CD}$} & Left ínsula & -29 & 24 & 15 & 825 & 4.18 & 0.007 \\
\hline & Left trigonal frontal gyrus & -32 & 24 & 15 & 147 & 3.90 & 0.020 \\
\hline
\end{tabular}

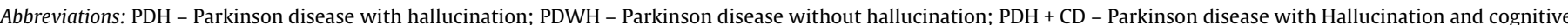
dysfunction; PDH - CD - Parkinson disease with Hallucination and without cognitive dysfunction.

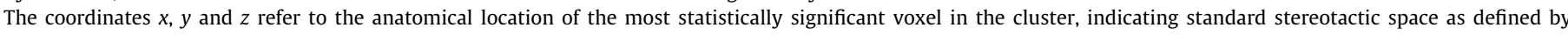
Tailarach and Tournoux.

* Values were obtained after small volume correction. 

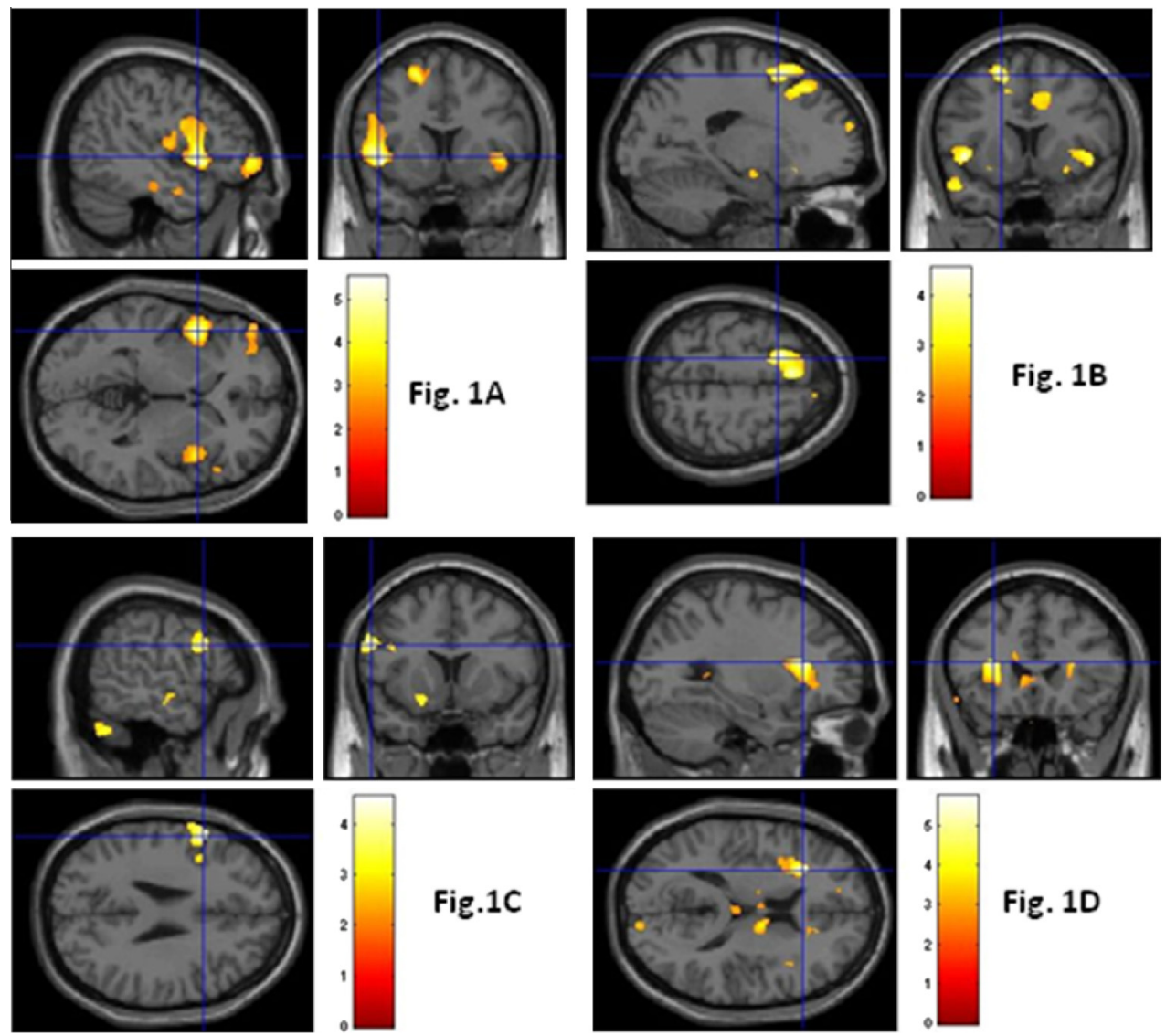

Fig.1C
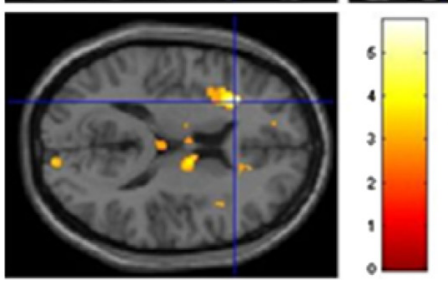

Fig. 1B

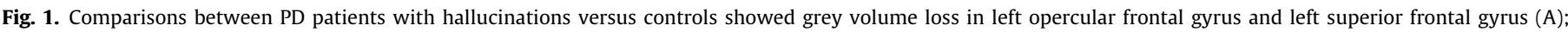

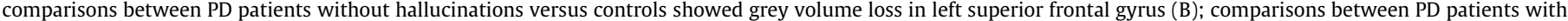

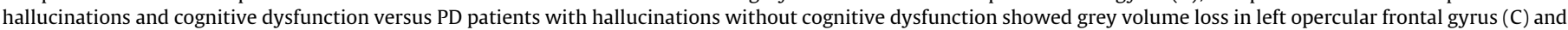

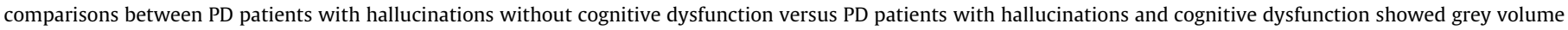
loss in left insula and left trigonal frontal gyrus (D).

showed hyperactivation in the right inferior frontal gyrus indicating an unbalance of hemispheres. The authors propose that a dysfunction of the frontal areas associated with the control of attention predisposes to $\mathrm{VHs}$ through an abnormal processing of relevant and irrelevant visual stimuli (Ramirez-Ruiz et al., 2008). Stebbins et al. showed that PD patients, with chronic VHs, respond to visual stimuli with greater frontal and subcortical activation and less visual cortical activation than non-hallucinating PD subjects. They propose that an abnormal cortex would result in deficiency of shifting visual circuitry from posterior to anterior regions. Therefore, impairment of networks involved in attention processes would have a role in the pathophysiology of visual hallucinations in PD (Stebbins et al., 2004). In corroboration to Stebbins findings, our findings indicate that reduced grey matter in the operculum is important for the presence of impaired circuitry and VHs. We propose that all these evidences favour the theory that hallucinations in PD are more related to a top-down abnormal process of images connected to a dysfunctional anterior cortex than a bottom-up abnormal function related to occipital cortex and other primarily afferent areas involved in the visual circuitry. Interestingly, similar to PD patients with VHs, a study of hallucinations in schizophrenia showed reduced connectivity for the right dorsolateral prefrontal cortex and reduced connectivity was also found to the left frontal operculum, extending into the anterior insula areas as well as the parietal opercular areas (Sommer et al., 2012).

Sanchez Castaneda et al. compared brain structural abnormalities in patients with VHs presenting either Diffuse Lewy Body (DLB) or PD. They showed that DLB subjects with VHs had greater grey matter loss than non-hallucinators, specifically in the right inferior frontal gyrus while PD patients with hallucinations had greater grey matter loss in the left orbitofrontal lobe. Furthermore, decreased volume in associative visual areas, namely left precuneus and inferior frontal lobe, correlated with VHs in DLB cases but not in PD patients. These findings are in concordance with our findings indicating that hallucinations in PD are more related to the involvement of anterior cortex than posterior associative cortex (Sanchez-Castaneda et al., 2010).

Ibarretxe-Bilbao et al. showed that patients with VH had widespread limbic, paralimbic and neocortical grey-matter loss, whereas in the PD without $\mathrm{VH}$ group, grey-matter loss was restricted to a small region in the frontal cortex and cerebellum (Ibarretxe-Bilbao et al., 2010). In this important prospective study, again, these alterations might have been connected to stages of disease severity and/ or interference with other factors such as cognitive alterations. In a previous study, comparing cases with VHs with and without dementia, Ibarretxe-Bilbao et al. showed that hallucinating PD patients had grey matter loss mainly confined to the hippocampal head while cases with dementia had grey matter loss of the entire hippocampus (Ibarretxe-Bilbao et al., 2008). In our study, differences between hippocampus were not found. These findings show that cortical deactivation of memory related structures can also be causal to VHs in PD. It can be hypothesised that altered connectivity between the hippocampus/parahippocampus with precuneus, parietal and occipital cortex also occur in hallucinating PD patients.

Limitations to this study must be acknowledged. Frequently, PD is a heterogeneous disorder with variable clinical manifestations. 
Cortical dysfunction, can be found or not during the illness adding to the complexity of this disease. Also, the study involved a small sample size. However, there is a relative paucity of literature regarding this topic. The present results contribute to understand the cerebral alterations in the course of the illness.

The observations reported here reinforce the concept that in PD, grey matter atrophy is precocious. Judging from the present findings, an abnormal frontal cortex, particularly left sided insula, frontal opercular, trigonal frontal gyrus and orbital frontal would make PD patients vulnerable to hallucinations. The operculum-insula network may be part of an imagery cortical release phenomenon and play an important role in the manifestation of hallucinations and cognitive dysfunction in PD. Our findings reinforce the theoretical concept of a top-down visual processing in the genesis of $\mathrm{VHs}$ in PD.

\section{Contributors}

VMSB designed the study. RLG and DGFT collected data. LB and ST analysed and interpreted data. All other authors gave comments on drafts of the paper.

\section{Acknowledgment}

\section{We wish to thank MCT/CNPq for partial support.}

\section{References}

Adachi, N., Watanabe, T., Matsuda, H., \& Onuma, T. (2000). Hyperperfusion in the lateral temporal cortex, the striatum and the thalamus during complex visual hallucinations: Single photon emission computed tomography findings in patients with Charles Bonnet syndrome. Psychiatry and Clinical Neurosciences, 54(2), 157-162.

Ashburner, J. (2007). A fast diffeomorphic image registration algorithm. Neuroimage, 38(1), 95-113.

Ashburner, J., \& Friston, K. J. (2005). Unified segmentation. Neuroimage, 26(3), 839-851.

Bar, M., Kassam, K. S., Ghuman, A. S., Boshyan, J., Schmid, A. M., Dale, A. M., et al. (2006). Top-down facilitation of visual recognition. Proceedings of the National Academy of Sciences USA, 103(2), 449-454.

Beck, A. T., \& Steer, R. A. (1984). Internal consistencies of the original and revised beck depression inventory. Journal of Clinical Psychology, 40(6), 1365-1367.

Bertolazi, A. N., Fagondes, S. C., Hoff, L. S., Dartora, E. G., Miozzo, I. C., de Barba, M. E., et al. (2011). Validation of the Brazilian Portuguese version of the Pittsburgh Sleep Quality Index. Sleep Medicine, 12(1), 70-75.

Bertolazi, A. N., Fagondes, S. C., Hoff, L. S., Pedro, V. D., Menna Barreto, S. S., \& Johns, M. W. (2009). Portuguese-language version of the Epworth sleepiness scale: Validation for use in Brazil. Jornal Brasileiro de Pneumologia, 35(9), 877-883.

Buysse, D. J., Reynolds, C. F., 3rd, Monk, T. H., Berman, S. R., \& Kupfer, D. J. (1989). The Pittsburgh Sleep Quality Index: A new instrument for psychiatric practice and research. Psychiatry Research, 28(2), 193-213.

Collerton, D., Perry, E., \& McKeith, I. (2005). Why people see things that are not there: a novel Perception and Attention Deficit model for recurrent complex visual hallucinations. The Behavioral and Brain Sciences, 28(6), 737-757. discussion 757-794.

Fahn, S., \& Elton, R. (1987). Unified Parkinson's disease rating scale. In S. Fahn, C. Marsden, D. Calne, \& M. Goldstein (Eds.), Recent developments in Parkinson's disease (pp. 153-163). Macmillan Health Care Information Florham Park.

Fenske, M. J., Aminoff, E., Gronau, N., \& Bar, M. (2006). Top-down facilitation of visual object recognition: Object-based and context-based contributions. Progress in Brain Research, 155, 3-21.

Folstein, M. F., Folstein, S. E., \& McHugh, P. R. (1975). "Mini-mental state”. A practical method for grading the cognitive state of patients for the clinician. Journal of Psychiatric Research, 12(3), 189-198.

Friston, K. J., Holmes, A. P., Poline, J. B., Grasby, P. J., Williams, S. C., Frackowiak, R. S., et al. (1995). Analysis of fMRI time-series revisited. Neuroimage, 2(1), 45-53.

Gibson, G., Mottram, P. G., Burn, D. J., Hindle, J. V., Landau, S., Samuel, M., et al. (2012). Frequency, prevalence, incidence and risk factors associated with visual hallucinations in a sample of patients with Parkinson's disease: A longitudinal 4-year study. International Journal of Geriatric Psychiatry, 28(6), 626-631.

Goetz, C. G., Vaughan, C. L., Goldman, J. G., \& Stebbins, G. T. (2013). I finally see what you see: Parkinson's disease visual hallucinations captured with functional neuroimaging. Movement Disorders, 29(1), 115-117.

Gomez-Esteban, J. C., Tijero, B., Somme, J., Ciordia, R., Berganzo, K., Rouco, I., et al. (2011). Impact of psychiatric symptoms and sleep disorders on the quality of life of patients with Parkinson's disease. Journal of Neurology, 258(3), 494-499.
Gorenstein, C., \& Andrade, L. (1996). Validation of a Portuguese version of the Beck Depression Inventory and the State-Trait Anxiety Inventory in Brazilian subjects. Brazilian Journal of Medical and Biological Research, 29(4), 453-457.

Harding, A. J., Broe, G. A., \& Halliday, G. M. (2002). Visual hallucinations in Lewy body disease relate to Lewy bodies in the temporal lobe. Brain, 125(Pt 2), 391-403.

Hoffstaedter, F., Grefkes, C., Roski, C., Caspers, S., Zilles, K., \& Eickhoff, S. B. (2014) Age-related decrease of functional connectivity additional to gray matter atrophy in a network for movement initiation. Brain Structure E' Function, [Epub ahead of print].

Huot, P., Johnston, T. H., Darr, T., Hazrati, L. N., Visanji, N. P., Pires, D., et al. (2010) Increased 5-HT2A receptors in the temporal cortex of parkinsonian patients with visual hallucinations. Movement Disorders, 25(10), 1399-1408.

Ibarretxe-Bilbao, N., Junque, C., Marti, M. J., \& Tolosa, E. (2011). Cerebral basis of visual hallucinations in Parkinson's disease: Structural and functional MRI studies. Journal of the Neurological Sciences, 310(1-2), 79-81.

Ibarretxe-Bilbao, N., Ramirez-Ruiz, B., Junque, C., Marti, M. J., Valldeoriola, F., Bargallo, N., et al. (2010). Differential progression of brain atrophy in Parkinson's disease with and without visual hallucinations. Journal of Neurology, Neurosurgery, and Psychiatry, 81(6), 650-657.

Ibarretxe-Bilbao, N., Ramirez-Ruiz, B., Tolosa, E., Marti, M. J., Valldeoriola, F., Bargallo, N., et al. (2008). Hippocampal head atrophy predominance in Parkinson's disease with hallucinations and with dementia. Journal of Neurology, 255(9), 1324-1331.

Janzen, J., Van't ent, D., Lemstra, A. W., Berendse, H. W., Barkhof, F., \& Foncke, E. M (2012). The pedunculopontine nucleus is related to visual hallucinations in Parkinson's disease: Preliminary results of a voxel-based morphometry study. Journal of Neurology, 259(1), 147-154.

Johns, M. W. (1991). A new method for measuring daytime sleepiness: The Epworth sleepiness scale. Sleep, 14(6), 540-545.

Kato, S., Watanabe, H., Senda, J., Hirayama, M., Ito, M., Atsuta, N., et al. (2012). Widespread cortical and subcortical brain atrophy in Parkinson's disease with excessive daytime sleepiness. Journal of Neurology, 259(2), 318-326.

Kazui, H., Ishii, R., Yoshida, T., Ikezawa, K., Takaya, M., Tokunaga, H., et al. (2009). Neuroimaging studies in patients with Charles Bonnet Syndrome. Psychogeriatrics, 9(2), 77-84.

Lewis, S. J., Shine, J. M., Duffy, S., Halliday, G., \& Naismith, S. L. (2012). Anterior cingulate integrity: Executive and neuropsychiatric features in Parkinson's disease. Movement Disorders, 27(10), 1262-1267.

Margis, R., Donis, K., Schonwald, S. V., Fagondes, S. C., Monte, T., Martin-Martinez, P., et al. (2009). Psychometric properties of the Parkinson's Disease Sleep ScaleBrazilian version. Parkinsonism \& Related Disorders, 15(7), 495-499.

Mechelli, A., Friston, K. J., Frackowiak, R. S., \& Price, C. J. (2005). Structural covariance in the human cortex. The Journal of Neuroscience, 25(36), 8303-8310.

Melzer, T. R., Watts, R., MacAskill, M. R., Pitcher, T. L., Livingston, L., Keenan, R. J., et al. (2012). Grey matter atrophy in cognitively impaired Parkinson's disease. Jounal of Neurology, Neurosurgery, and Psychiatry, 83(2), 188-194.

Memoria, C. M., Yassuda, M. S., Nakano, E. Y., \& Forlenza, O. V. (2013). Brief screening for mild cognitive impairment: Validation of the Brazilian version of the Montreal cognitive assessment. International Journal of Geriatric Psychiatry, 28(1), 34-40.

Meppelink, A. M., de Jong, B. M., Renken, R., Leenders, K. L., Cornelissen, F. W., \& van Laar, T. (2009). Impaired visual processing preceding image recognition in Parkinson's disease patients with visual hallucinations. Brain, 132(Pt 11), 2980-2993.

Naismith, S. L., \& Lewis, S. J. (2011). “DASH” symptoms in patients with Parkinson's disease: Red flags for early cognitive decline. Journal of Clinical Neuroscience, 18(3), 352-355.

Papapetropoulos, S., McCorquodale, D. S., Gonzalez, J., Jean-Gilles, L., \& Mash, D. C. (2006). Cortical and amygdalar Lewy body burden in Parkinson's disease patients with visual hallucinations. Parkinsonism \& Related Disorders, 12(4), 253-256.

Ramirez-Ruiz, B., Marti, M. J., Tolosa, E., Falcon, C., Bargallo, N., Valldeoriola, F., et al (2008). Brain response to complex visual stimuli in Parkinson's patients with hallucinations: A functional magnetic resonance imaging study. Movement Disorders, 23(16), 2335-2343.

Ramirez-Ruiz, B., Marti, M. J., Tolosa, E., Gimenez, M., Bargallo, N., Valldeoriola, F., et al. (2007). Cerebral atrophy in Parkinson's disease patients with visual hallucinations. European Journal of Neurology, 14(7), 750-756.

Rodriguez-Violante, M., Cervantes-Arriaga, A., Villar-Velarde, A., \& Corona, T. (2010). Prevalence of non-motor dysfunction among Parkinson's disease patients from a tertiary referral center in Mexico City. Clinical Neurology and Neurosurgery, $112(10), 883-885$.

Salmond, C. H., Ashburner, J., Vargha-Khadem, F., Connelly, A., Gadian, D. G., \& Friston, K. J. (2002). Distributional assumptions in voxel-based morphometry. Neuroimage, 17(2), 1027-1030.

Sanchez-Castaneda, C., Rene, R., Ramirez-Ruiz, B., Campdelacreu, J., Gascon, J. Falcon, C., et al. (2010). Frontal and associative visual areas related to visual hallucinations in dementia with Lewy bodies and Parkinson's disease with dementia. Movement Disorders, 25(5), 615-622.

Sommer, I. E., Clos, M., Meijering, A. L., Diederen, K. M., \& Eickhoff, S. B. (2012). Resting state functional connectivity in patients with chronic hallucinations. PLoS One, 7(9), e43516.

Stebbins, G. T., Goetz, C. G., Carrillo, M. C., Bangen, K. J., Turner, D. A., Glover, G. H., et al. (2004). Altered cortical visual processing in PD with hallucinations: An fMRI study. Neurology, 63(8), 1409-1416. 
Tse, W., Liu, Y., Barthlen, G. M., Halbig, T. D., Tolgyesi, S. V., Gracies, J. M., et al. (2005). Clinical usefulness of the Parkinson's disease sleep scale. Parkinsonism E Related Disorders, 11(5), 317-321.

Vaden, K. I., Jr., Kuchinsky, S. E., Cute, S. L., Ahlstrom, J. B., Dubno, J. R., \& Eckert, M. A (2013). The cingulo-opercular network provides word-recognition benefit. The Journal of Neuroscience, 33(48), 18979-18986.
Wakamori, T., Agari, T., Yasuhara, T., Kameda, M., Kondo, A., Shinko, A., et al. (2014). Cognitive functions in Parkinson's disease: Relation to disease severity and hallucination. Parkinsonism \& Related Disorders, [Epub ahead of print].

Williams, D. R., \& Lees, A. J. (2005). Visual hallucinations in the diagnosis of idiopathic Parkinson's disease: A retrospective autopsy study. Lancet Neurology, 4(10), 605-610. 